\title{
Design of 2-4 GHz Equalizers for the Antiproton Accumulator Stacktail System
}

\author{
C. Deibele
}

Abstract: The antiproton source at Fermilab requires storage of antiprotons during the production of antiprotons. A fundamental part of the storage process involves stochastic cooling, which requires that the frequency spectrum from the pickups has notches at the revolution frequency and harmonics of the revolution frequency of the antiprotons in the storage ring. ${ }^{1}{ }^{2}$ A system has been developed for broadband notches but suffers from dispersion. The dispersion inhibits the cooling process and therefore an equalizer is required. The process for designing the equalizers is described and results shown.

\section{Introduction and Theory of the Notch Network}

It is desired in the antiproton source at Fermilab to have a repetitive notch filter network where notches fall at specific harmonic frequencies of the revolution frequency of the. A schematic of an idealized repetitive notch filter network is shown in Fig. 1. In the schematic of

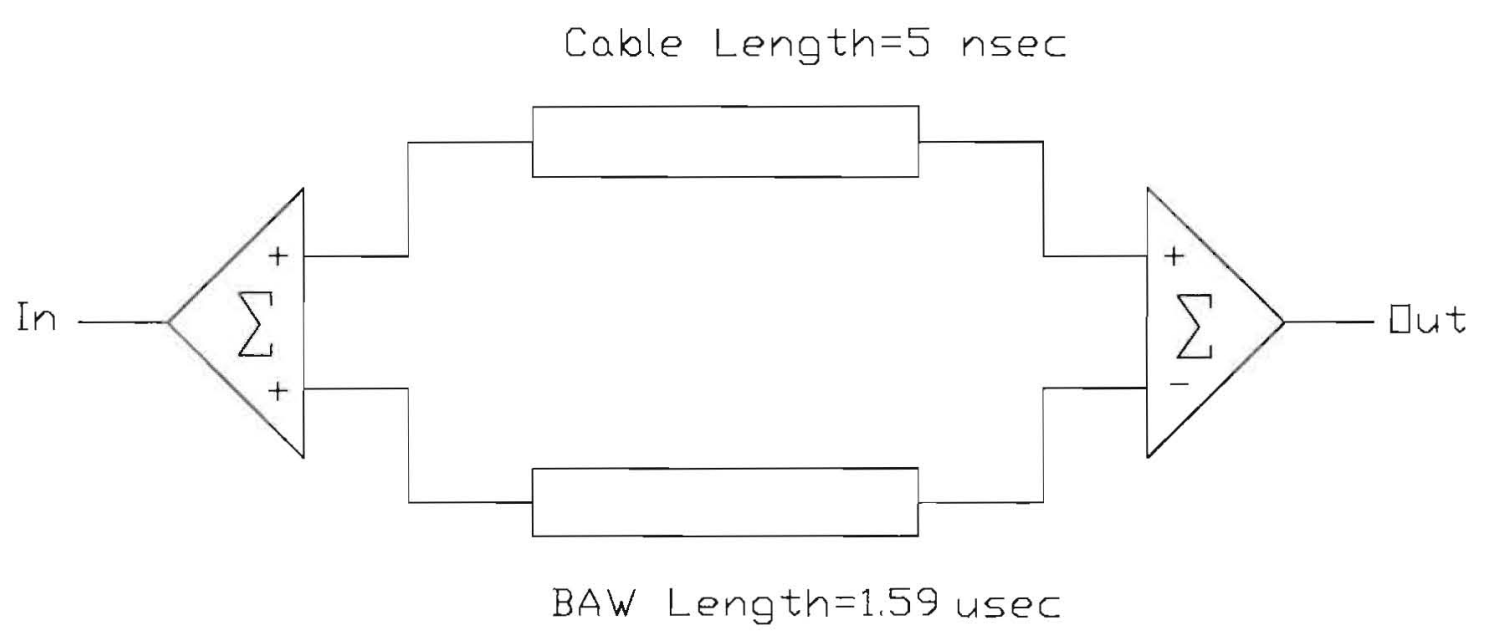

Figure 1. A schematic of a repetitive notch filter network. The separation of notches is determined by the time difference between each leg of the filter.

\footnotetext{
${ }^{1}$ R. Pasquinelli, "Bulk Acoustic Wave (BAW) Devices for Stochastic Coolig Notch Filters," IEEE Nucl Sci, May 1991.

${ }^{2}$ R. Pasquinelli, "Superconducting Notch Filters for the Fermilab Antiproton Source,"
} 
Fig. 1 each cable is assumed to have no dispersion and no loss. A bulk acoustic wave (BAW) device is a compact network which has a specified delay over the bandwidth of interest. The output of an ideal repetitive notch filter is as follows:

$$
S_{21}(\omega)=e^{i \omega t_{1}}-e^{i \omega t_{2}}
$$

Using familiar trigonometric identities, Eq. 1 may be rewritten into Eq. 2

$$
e^{i \omega t_{1}}-e^{i \omega t_{2}}=2 i \sin \left(\frac{1}{2} \omega\left(t_{2}-t_{1}\right)\right) e^{i \omega\left(t_{1}+t_{2}\right)} .
$$

Equation 2 specifically shows the frequencies where the notches lie for the notch filter. Each notch frequency is determined by the time difference between each leg and each notch can be determined by the relationship

$$
\omega_{\text {notch }}=\frac{2 m \pi}{t_{2}-t_{1}}, \quad m=0,1,2, \ldots
$$

A plot depicting the frequency response of the notch filter network is shown in Fig. 2. A salient point to note about this equation is that notches lay at DC and at multiples of $\frac{2 \pi}{t_{2}-t_{1}}$.

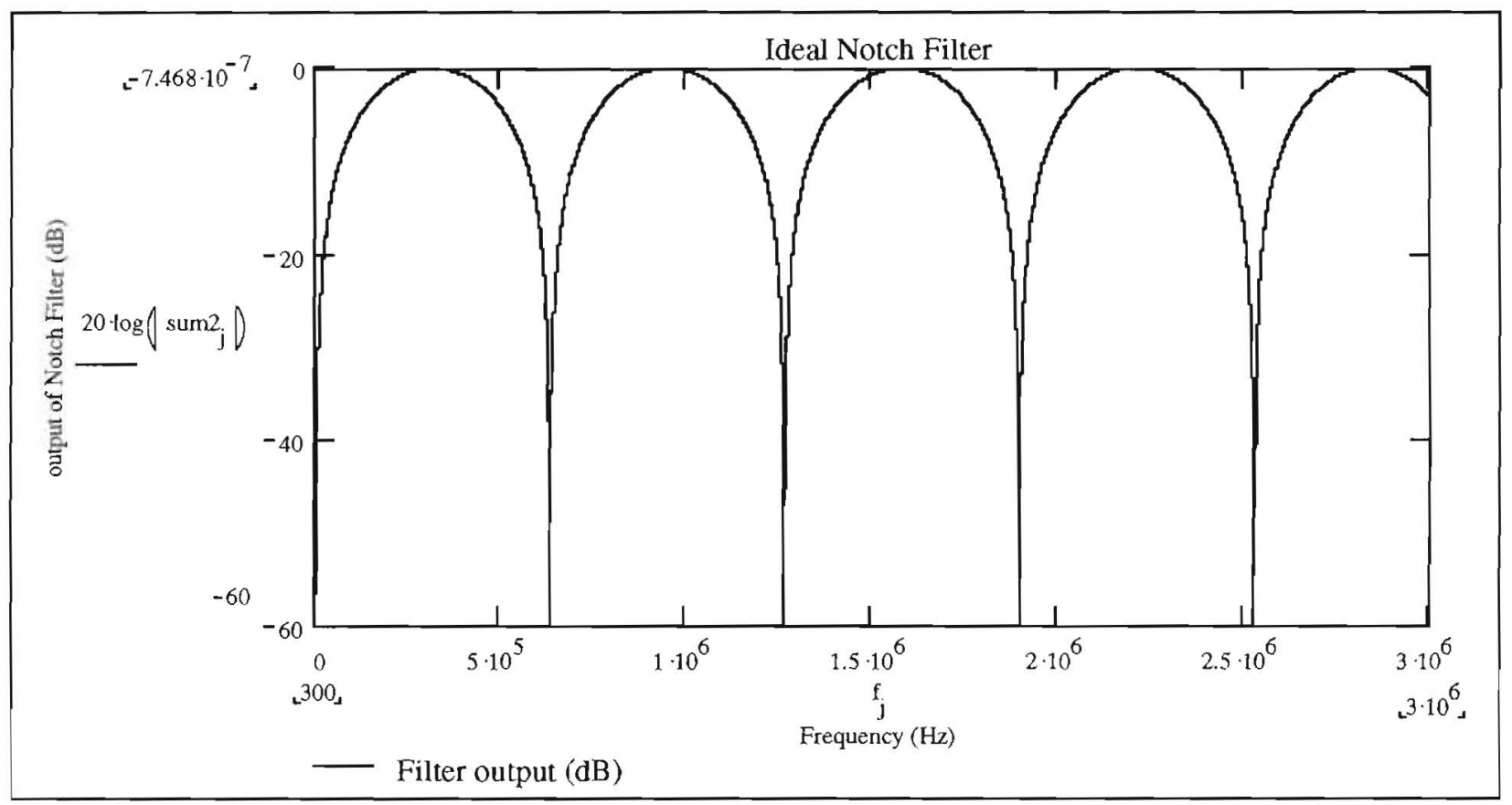

Figure 2. The plot of the ideal notch filter network depicted in Fig. 1. 
Imagine a scenario where the long leg has an additional broadband phase. This changes the results of Eq. 1 to $s_{21}(\omega)=e^{i \omega t_{1}}-e^{i \omega t_{1}+i \phi}$. After a few trigonometric substitutions results in a form similar to Eq. 2:

$$
e^{i \omega t_{1}}-e^{i \omega t_{2}+i \phi}=2 i \sin \left(\frac{1}{2} \omega\left(t_{2}-t_{1}\right)+\frac{\phi}{2}\right) e^{i \omega\left(t_{1}+t_{2}+\frac{\phi}{2}\right)} .
$$

This equation clearly shows that the frequencies of the notches do not at exist at any harmonic because of the additional phase in the sine term. It does show that the frequency spacing from notch to notch can be a harmonic. A plot of two systems, one with a small broad band phase, and the data of Fig. 2 is presented in Fig. 3. It is clear from this plot that additional phase changes the frequency of the notch, but not the spacing of the notch. It is desired for the stacktail notch filter network that $\phi$ is as close to zero as possible.

In any physical system a non-ideal situation exists regarding each component of the

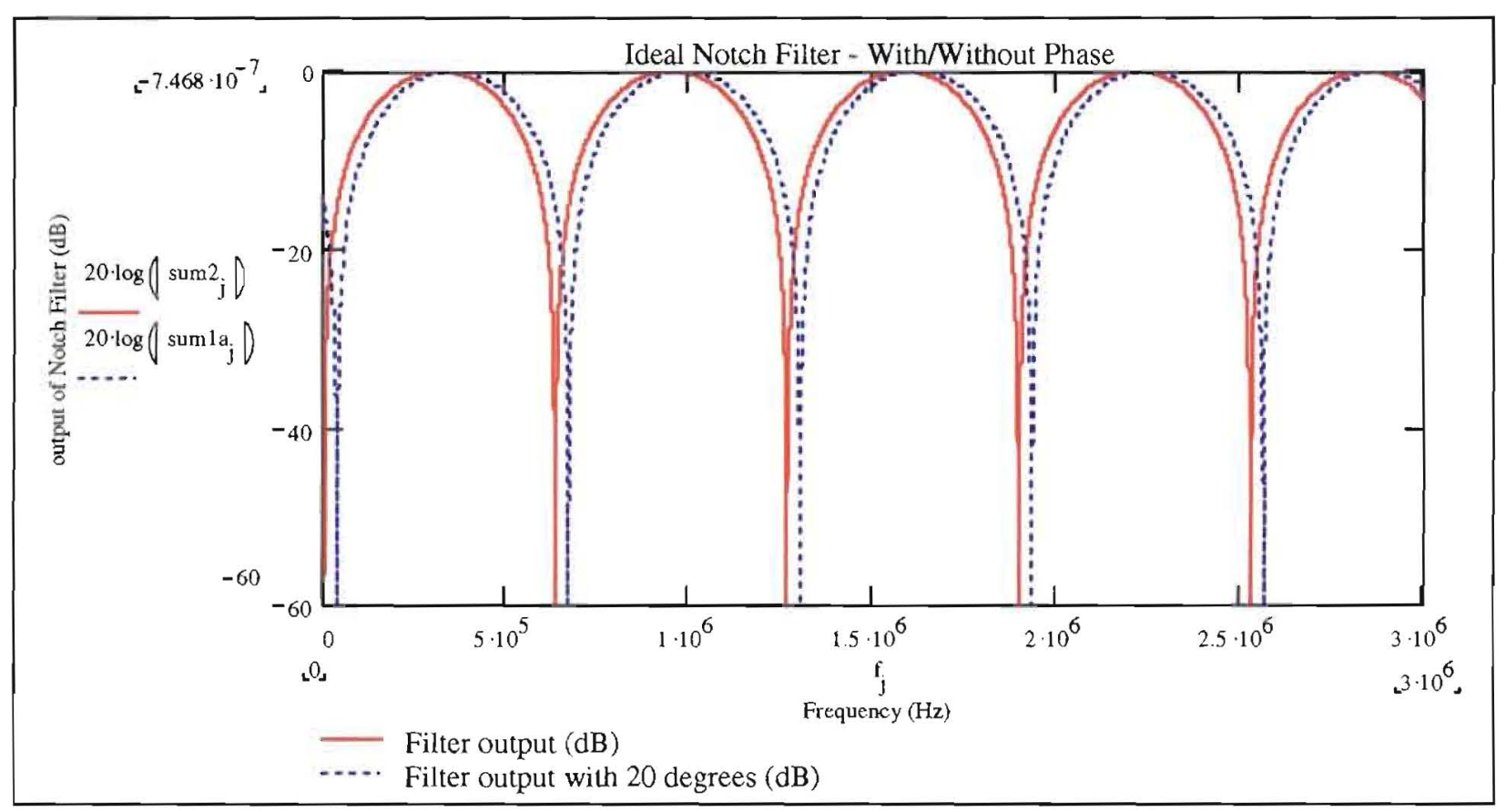

Figure 3. A plot of two ideal notch filters, one without phase, the other with $20^{\circ}$ of broadband phase added. The notch spacing stays the same, but the frequency of the notch locations changes. 
network of Fig. 1. A short list of ideal assumptions made in the schematic of Fig. 1 are as follows:

- The $3 \mathrm{~dB}$ splitters do not distribute the energy and phase of the incoming signal equally between each leg.

- The BAW suffers from dispersion and nonuniform amplitude over the band of interest.

- The BAW requires amplifiers for magnitude matching. These amplifiers add dispersion and nonuniform amplitude.

- The $180^{\circ}$ hybrid has dispersion and nonuniform magnitude match

- $\quad$ Additional components which are not shown (i.e. trombone section, attenuators, cables, and switches) add additional dispersion and amplitude fluctuations.

To accommodate the non-idealities of the filter, an equalizer is required to match the individual legs the filter.

\section{The Schiffmann Phase Shifter Theory and Its Application to an Equalizer}

Correcting the phase dispersion of an element can be accomplished through the use of Schiffmann phase shifters (SPS). An SPS is, quite simply, a coupled line structure with two ports shorted together. A typical example of an SPS is shown in Fig. 4. Assuming that the SPS is matched correctly and reflections are small (on the order of $-20 \mathrm{~dB}$ or smaller), the transmission coefficient through an SPS is

$$
\mathrm{s}_{21}(\omega)=\mathrm{e}^{-\mathrm{i} \beta\left(21_{\text {coup }}+\mathrm{i}_{\text {teece }}\right)+\mathrm{i} C(\text { coupling }) \sin \left(\frac{\omega \mathrm{I}_{\text {coup }}}{\pi \mathrm{c}}\right)} .
$$

The factor $\mathrm{C}$ (coupling) is a constant and is a function of the coupling from one conductor to the other, $\mathrm{c}$ is a constant and is the speed of light on the coupling structure, $\beta$ is the wave-number, $l_{\text {coup }}$ is the length of the coupled line section, and $l_{\text {feed }}$ is the length of the feed lines leading to/from the SPS.

The SPS is clearly a phase dispersive structure because of the existence of an additional term in the exponential, namely $\mathrm{iC}$ (coupling) $\sin \left(\frac{\omega \mathrm{l}_{\text {coup }}}{\pi \mathrm{c}}\right)$ of the transmission coefficient. The 
positive part of the

additional

dispersion is that it

is completely

controllable. This

phase dispersion

term can be used to

offset any band

limited phase

dispersion in

almost any given

Figure 4. An example of an SPS. This particular SPS is constructed using system. This can a microstrip or stripline configuration.

be shown rather easily using a Fourier sine series. It has been shown ${ }^{3}$ that any function can be represented using either a Fourier cosine series, a Fourier sine series, or a Fourier complex exponential series. Since all physical systems, the SPS included, have an odd phase symmetry about frequency $=0$, it is natural and obvious to choose a Fourier sine series.

Realizing a particular equalizing network requires cascading SPS's. Cascading several SPS's results in the following transmission:

$$
S_{21-\text { cascaded }}(\omega)=\mathrm{e}^{\sum^{\mathrm{n}}-\mathrm{i} \beta 2 \mathrm{l}_{\mathrm{n}}+\mathrm{i} C_{\mathrm{n}} \sin \left(\frac{\omega \mathrm{l}_{\mathrm{n}}}{\pi \mathrm{c}}\right)}
$$

${ }^{3}$ See, for example, R. Churchill, J. Brown, "Fourier Series and Boundary Value Problems," Fourth Edition, Mc-Graw Hill Book Company, 1987. 
Careful choice of the $\mathrm{C}_{\mathrm{n}}$ 's and $\mathrm{l}_{\mathrm{n}}$ 's can produce any desired phase response. Equation 4 does not include the effects of any feed lines.

\section{Design Example}

Imagine that a system exists with phase dispersion. It is desired to take the phase dispersion out of the system by designing an equalizer. The system has a frequency response of

$$
\mathrm{s}_{21}(\omega)=\mathrm{A}(\omega) \mathrm{e}^{-\mathrm{i} \omega \mathrm{t}_{\mathrm{dclay}}+\mathrm{i} \varphi(\omega)}
$$

In Eq. $5, \mathrm{~A}(\omega)$ is a real function of frequency, $\mathrm{t}_{\text {delay }}$ is a constant, and $\varphi(\omega)$ is a real function of frequency. The system has a center frequency of $\mathrm{f}_{\mathrm{o}} \mathrm{Hz}$ and a bandwidth of $\mathrm{B} \mathrm{Hz}$. To make this example easier, assume that $\mathrm{f}_{0}-\mathrm{B}<0$. This assumption can be easily removed by using the periodicity of the Fourier sine series and will not be discussed in this paper.

Using linear system theory, cascading a low reflection system to the system described in Eq. 5 can be realized using the schematic of Fig. 5 .

The output of the cascaded network of Fig. 5 is simply the multiplication

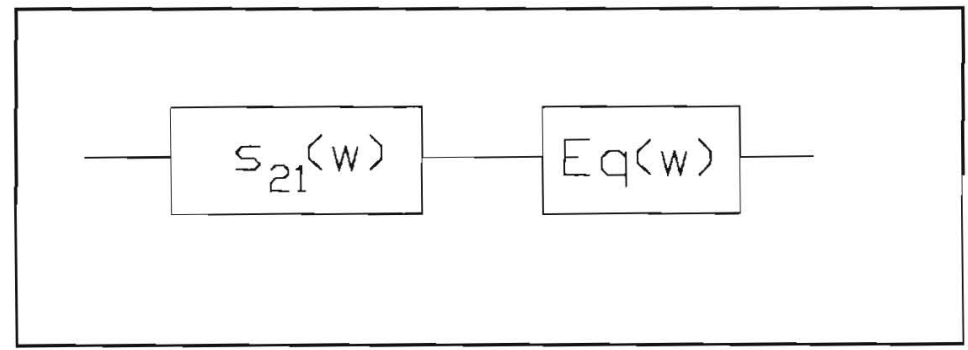

Figure 5. Placement of an equalizing network for the dispersive system $s_{21}(\omega)$.

of the two networks. This multiplication results in the following frequency response of the network:

$$
A(\omega) e^{-i \omega t_{\text {delay }}+i \varphi(\omega)+\sum_{n}-i \beta 21_{n}+i C_{n} \sin \left(\frac{\omega l_{n}}{\pi c}\right)} .
$$

The linear phase elements do not add to the dispersion calculation. A network with zero phase dispersion therefore requires that

$$
i \varphi(\omega)=-\sum_{n} i_{n} \sin \left(\frac{\omega l_{n}}{\pi c}\right) .
$$


Equation 7 looks suspiciously like a Fourier sine series. Using Eq. 7 in a Fourier sine series requires that $\frac{\left(\omega_{0}+\pi \mathrm{B}\right) \mathrm{l}_{1}}{\pi \mathrm{c}}>\pi$. This requirement implies that a lower limit for the length of each SPS exists. This requirement is important for the series to converge adequately.

Furthermore, choosing an $l_{1}$ less than this requirement would invalidate the assumptions of using a Fourier sine series. If one were to choose $1_{1}=\frac{\pi^{2} \mathrm{c}}{\omega_{0}+\pi \mathrm{B}}$ then the boundary condition at the upper regime of the frequency band would not be satisfied since the sine functions in the series are identically zero. This constraint is related to the Gibbs phenomenon, and therefore a choice of $\frac{\left(\omega_{0}+\pi \mathrm{B}\right) 1_{1}}{\pi \mathrm{c}}=1.2 \pi$ is a safe minimum choice for $1_{1}$. An equation for the remaining $\mathrm{l}_{n}$ 's is

$$
\mathrm{l}_{\mathrm{n}}=\mathrm{nl}_{1} \quad \mathrm{n}=2,3,4, \ldots
$$

\section{Application to the Stacktail System}

The 2-4 GHz stacktail system in the antiproton source required the design of equalizers because of phase dispersion. The method for designing the first filter will be described and results shown.

For the notch filter to function correctly, it is required that the long leg (or leg with the BAW), has an amplitude match of $\pm 1 \mathrm{~dB}$ and a $\pm 10^{\circ}$ match to the short leg. It was desired to accommodate all the nonlinear elements into the design of the equalizer. To ease the design, it was decided to calibrate a vector network analyzer (HP 8522) along the short leg and equalize the long leg to the short leg. This effectively made the $t_{\text {detay }}$ term equal to zero and the $A(\omega)$ term equal to 1 in Eq. 6. From that point, it was only necessary to determine whether the long leg required magnitude equalization and/or phase equalization.

The magnitude of the long leg is shown in Fig. 6. Since the in-band magnitude is within specifications, then it was only necessary to examine the phase response. The phase response is 
shown in Fig. 7.

The phase of the

long leg was

desired to be $180^{\circ}$

and the data shown

in Fig. 7 show that

the first $300 \mathrm{MHz}$

and the last 50

$\mathrm{MHz}$ had too much

phase dispersion.

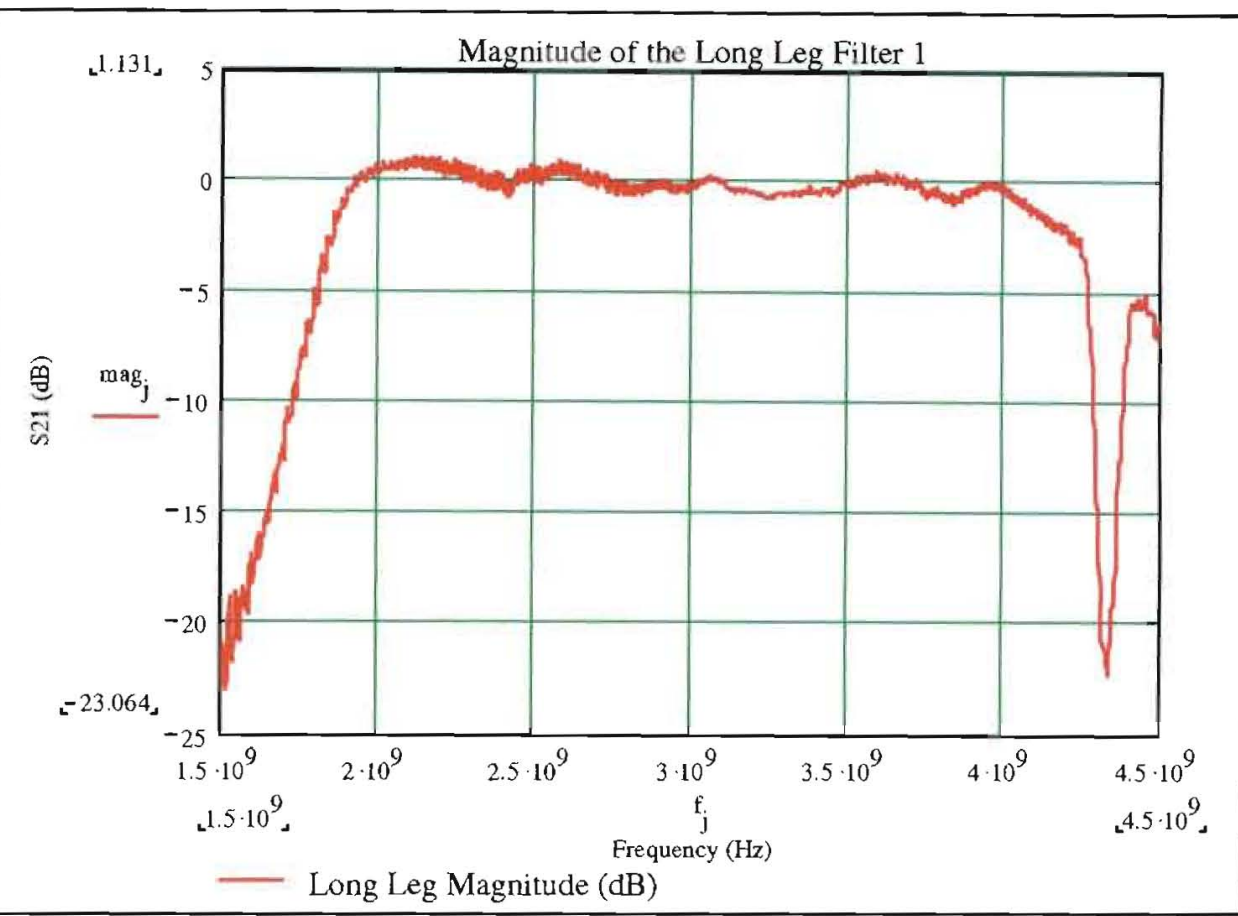

Figure 6. The magnitude of the long leg response to the $2-4 \mathrm{GHz}$ stacktail

In fact, at the band system. Since the magnitude requirements are satisfied (i.e. $+/-1 \mathrm{~dB}$ ), then only phase equalization is required.

edge at $2 \mathrm{GHz}$, the phase was off by as

much as $45^{\circ}$. A

series of SPS's was

used to equalize the

phase to the desired

response.

The

realization of the

equalizer was

performed using

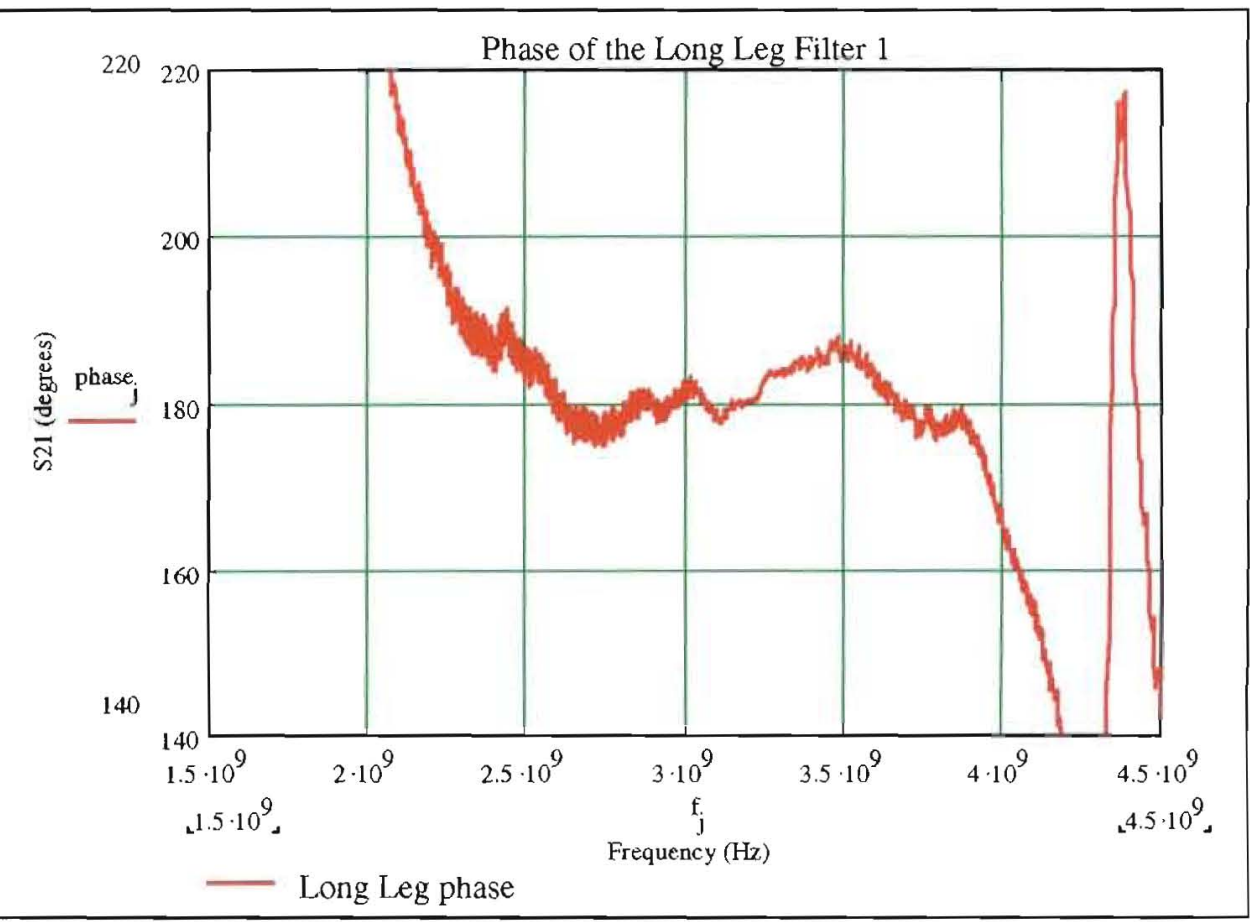

Figure 7. The dispersive phase response of the long leg of the $2-4 \mathrm{GHz}$ stacktail system. It was required to equalize the phase to 180 degrees. 


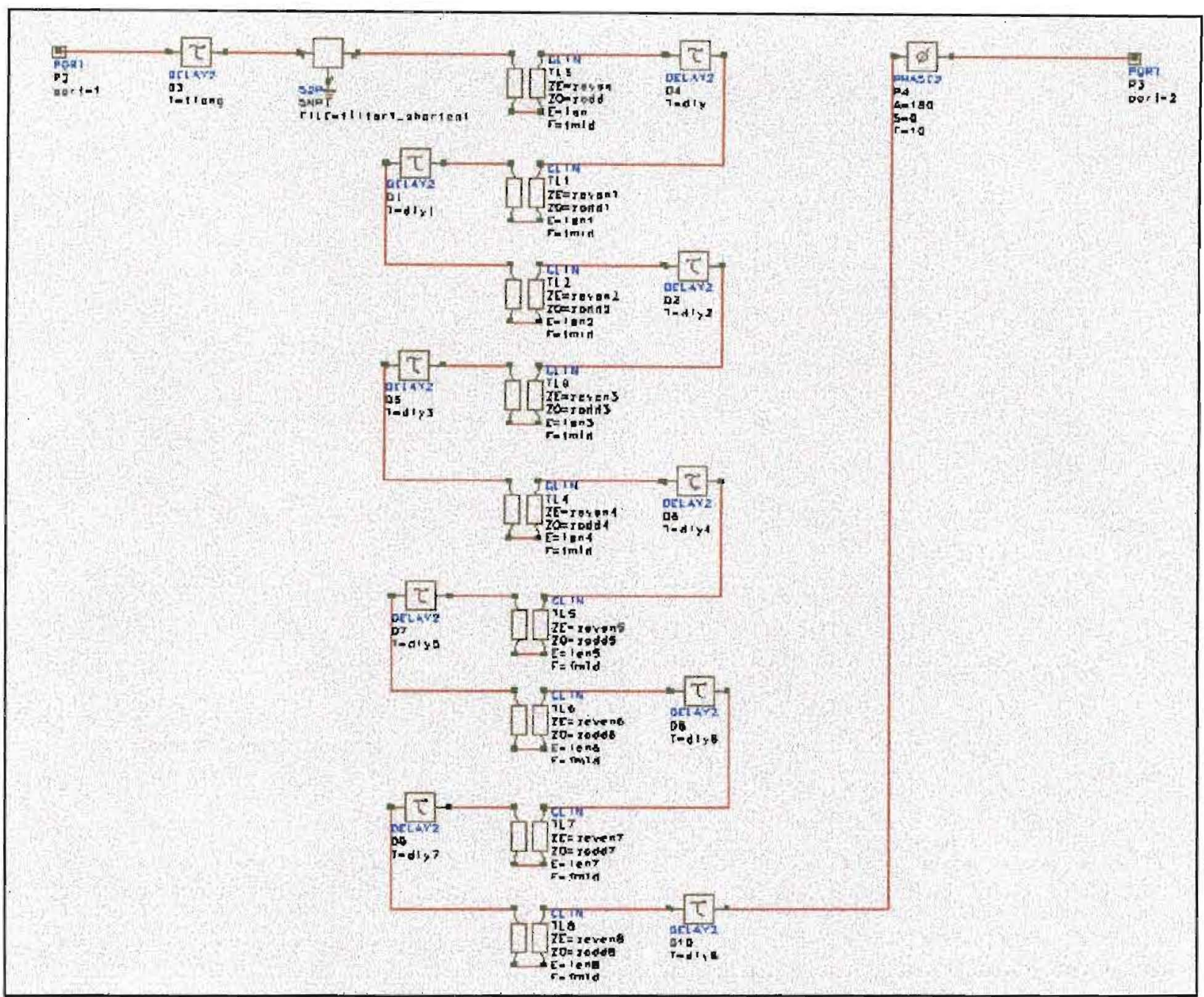

Figure 8. A picture of the circuit for designing the equalizer from Series IV.

Series IV (a microwave circuit simulator developed by the former Hewlett Packard corporation, presently Agilent Technologies). A picture of the circuit is shown in Fig. 8. The first element adjusts the delay from the measured data and is equivalent taking out the $\mathrm{e}^{-i \omega_{\text {delay }}}$ term in Eq. 5 . The next item is the data from the network analyzer. Then one sees a series of coupled lines and delay elements. The delay elements for each coupled line take out the $\mathrm{e}^{-\mathrm{i} 2 \beta \mathrm{l}_{\mathrm{n}}}$ in Eq. 6 . The even and odd impedances of each coupled line are designed to have a $50 \Omega$ match. Finally, an additional $180^{\circ}$ phase shifter is added for the optimization so that the total phase through the 
system is $0^{\circ}$. This is

important since

small fluctuations

about $180^{\circ}$ result in

a phase being either

$+180^{\circ}$ or $-180^{\circ}$. A

plot of the circuit

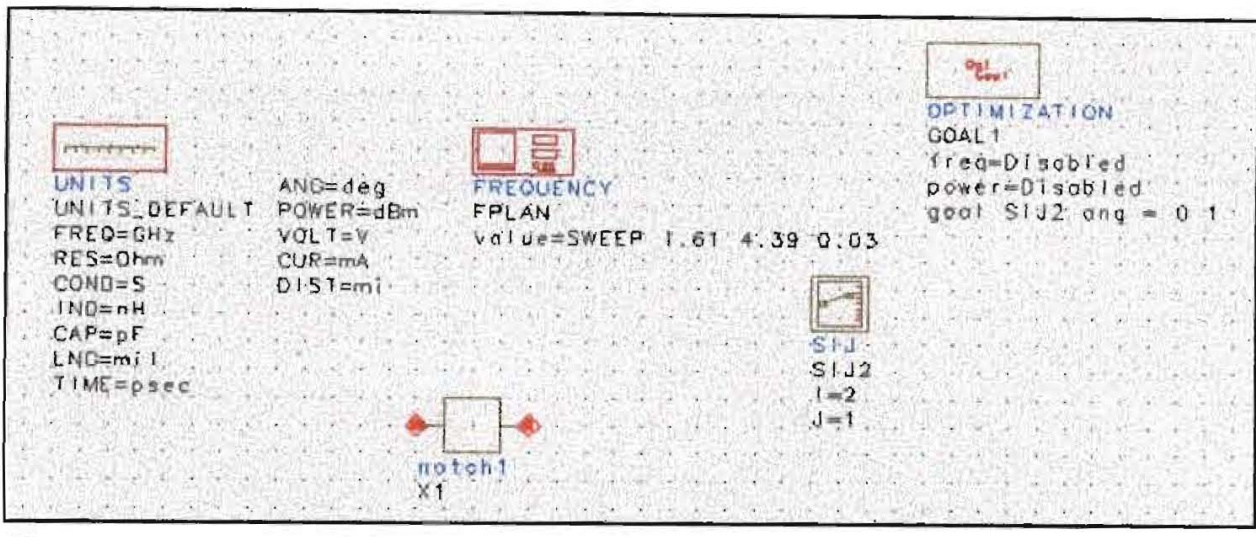

Figure 9. A picture of the test bench for the circuit in Fig. 7. The optimization goal of the total phase being zero degrees is also listed.

test bench with the appropriate optimization goal is shown in Fig. 9. It was important to include frequencies in the optimization out of the band so that the phase at the immediate band edges is flat.

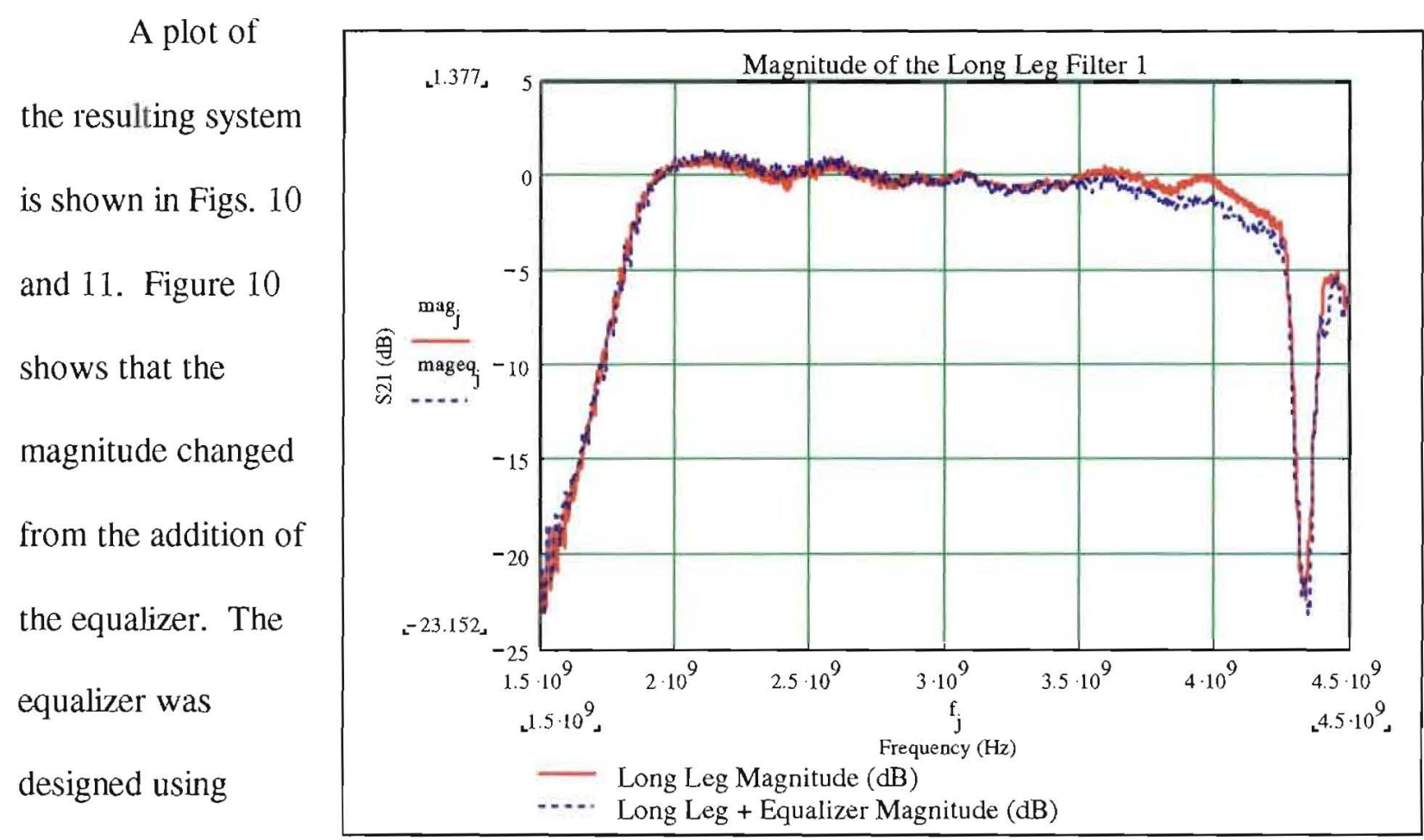

stripline and

Figure 10. A plot of the long leg - with and without the equalizer. Note that the SPS in theory does not change the magnitude of the long leg. The therefore the magnitude variation added by the SPS is a result of dielectric losses in the equalizer. 
magnitude variation

is a result of

ielectric losses in

the equalizer and

could not be

avoided. The

magnitude variation

can be minimized

by using another

circuit topology

(i.e. microstrip) or

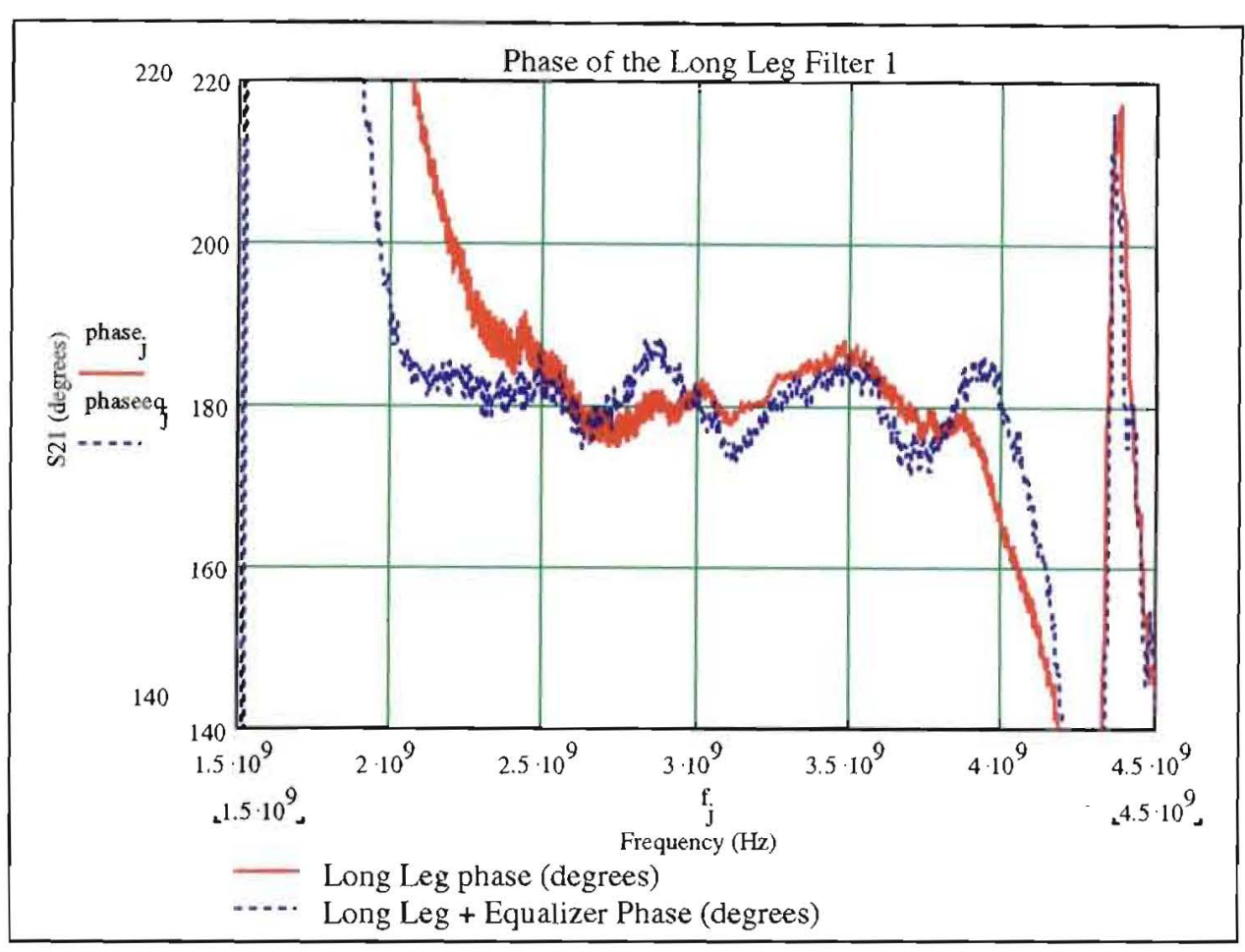

Figure 11. The phase of the long leg with and without the equalizer.

less lossy dielectric. Figure 11 shows that the phase goal was achieved.

A picture of the actual board layout is shown in Fig. 12. Careful examination of Fig. 12 shows that a true sine series was not used. While a sine series will always produce the desired result, it may require more terms than allowing a slight length variation in each individual SPS.

To design each SPS, Ensemble (a moment method program by Ansoft Corporation) was employed to tweak the design parameters by Series IV. Ensemble was not capable of predicting the group delay through each SPS. Additionally, Ensemble could not predict the $\mathrm{C}_{\mathrm{n}}$ terms shown in Eq. 4. Some of the $\mathrm{C}_{\mathrm{n}}$ terms were off by as much as $80 \%$. Consequently Ensemble could only be used for designing how the coupled lines are connected together (see for example Fig. 4) and how the mitered bend for launching the wave into the SPS should be designed so that a minimum reflection coefficient is observed. And finally, since Ensemble could not predict with any 


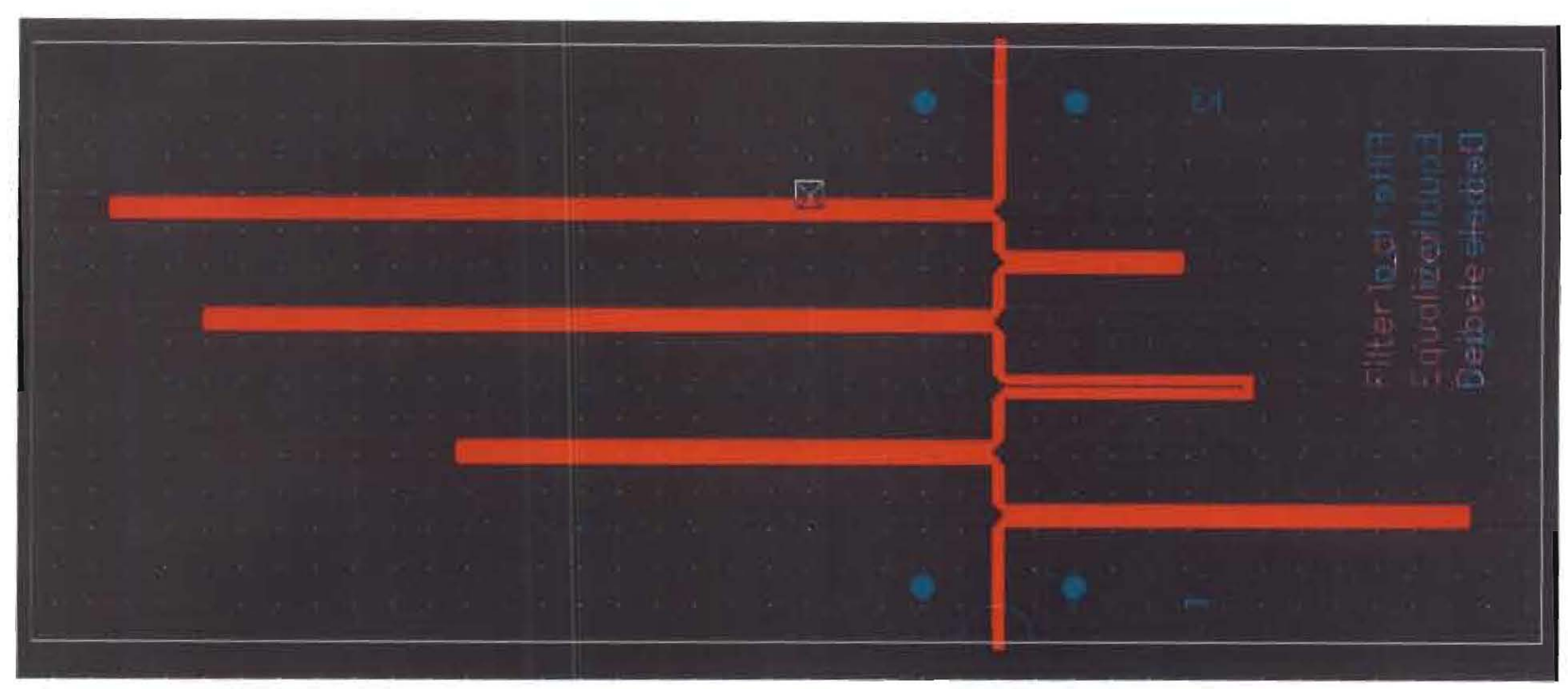

Figure 12. A picture of the equalizer layout. The circuit board dimensions are $0.095 "$ x $10^{\prime \prime}$ x 4". The circuit was manufactured on a substrate by Arlon and is a CUCLAD LX-04503355.005 dielectric constant of the board is 2.32 .

accuracy the $C_{n}$ terms the line spacing between each coupled line predicted by Series IV was used.

\section{Conclusion}

The stacktail 2-4 GHz system required the use of equalizers for flattening the phase response of the long leg. Use of SPS can produce excellent results. Using a collage of Fourier series mathematics, Series IV optimization, and Ensemble produced results within specification.

\section{Acknowledgements}

I would like to thank Ralph Pasquinelli, Dave McGinnis, Paul Derwent, Dave Vander Muelen, and Ed Cullerton for our enlightening discussions about the theory of the stochastic cooling system. I also with to thank Wes Mueller, Mark Dilday, Ed Cullerton, Don Poll, and Ozark Circuits for their technical expertise in helping in the manufacture of parts for the equalizer. 